\title{
LE TUNNEL HYDRODYNAMIQUE DE L'UNIVERSITÉ DE GRENOBLE
}

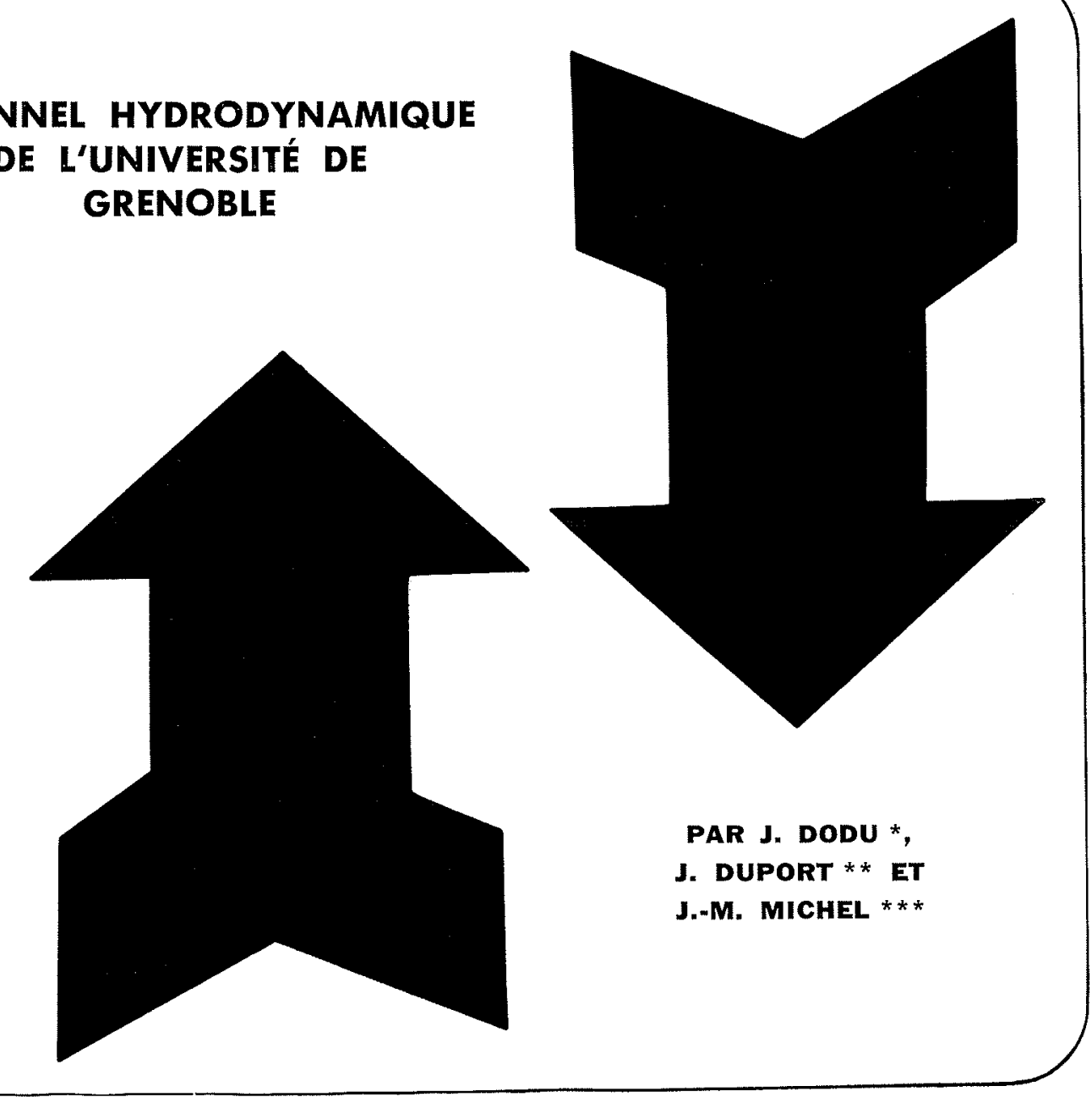

Le tunnel hydrodynamique des Laboratoires de Mécanique des Fluides de l'Université de Grenoble prend place à côté des moyens d'essais utilisés dans le domaine des écoulements rapides cavitants et supercavitants $\left(^{1}\right)$ :

- bassins de carènes permettant la réalisation de grandes dimensions d'écoulement et la simulalion de la houle;

- bassins de carènes à pression réglable dans lesquels on peut réaliser la cavitation spontanée; - tunnels à rafale où l'on peut obtenir soit des nombres de cavitation très petits, soit la simulation des grandes profondeurs;

remorquage de profil sur lac naturel offrant à peu de frais la possibilité de réaliser de grands nombres de Reynolds avec une très faible turbulence.

Cet équipement a été projeté sous l'impulsion de la Direction des Recherches et des Moyens d'Essais (D.R.M.E.) et on s'est arrêté au choix d'un tunnel en circuit fermé pour disposer à un coût relativement modéré de bonnes conditions d'observation des écoulements autour de profils cavitants et supercavitants et de facilités de mesures dans ces écoulements.

* Professeur a la Faculté des Sciences de Grenoble.

* Directeur de la Division des Techniques Industrielles à la SOGREAH.

** Chercheur aux Laboratoires de Mécanique des fluides de l'Université de Grenoble.

(1) Symposium on cavitation research facilities and techniques, Philadelphie (mai 1964).
PAR J. DODU *

J. DUPORT * * ET

J.-M. MICHEL ***
A Grenoble, la constitution d'une équipe de recherche de cavitation s'est justifiée par la présence d'un laboratoire universitaire engagé dans la recherche théorique et expérimentale en mécanique et en mécanique des fluides et de la SOGREAH. établissement industriel de recherche et d'ingéniérie, dans le domaine du génie hydraulique.

Les premières décisions se sont matérialisées en 1964 par la conclusion d'un contrat pour la construction d'un tunnel hydrodynamique pour étude de profils. Le financement de la construction était assuré par la Direction des Recherches et Moyens d'Essais, et une partie importante de l'exécution était sous-traitée par la SOGREAH. Les études ont été achevées en 1965, et la mise en eau a eu lieu en juin 1966. Les essais de réception et diverses améliorations ont été réalisés en 1967.

Caractéristiques essentielles

En ce qui concerne la géométrie, le tunnel de Grenoble peut recevoir des veines interchangeables. Celles-ci peuvent être en charge ou à surface libre. Les veines à surface libre sont alors enfermées dans un caisson étanche qui permet de fixer la valeur de la pression au-dessus de la surface. Toutes les veines ont 2,50 m de longueur; leurs dimensions maximales sont: diamètre $400 \mathrm{~mm}$ pour la veine de section circulaire et $250 \times 400 \mathrm{~mm}$ pour les veines de section rectangulaire; l'aire de la section est dans chaque cas voisine de $10 \mathrm{dm}^{2}$. 


\section{J. DODU, J. DUPORT et J.-M. MICHEL}

Le débit maximal a été fixé à $0,6 \mathrm{~m}^{3} / \mathrm{s}$. La vitesse maximale peut atteindre $12,25 \mathrm{~m} / \mathrm{s}$ avec la veine actuellement réalisée (largeur $175 \mathrm{~mm}$, hauteur $280 \mathrm{~mm}$ ). L'installation prochaine du tunnel dans les nouveaux locaux des Laboratoires au Domaine Universitaire de Saint-Martin-d'Hères permettra de porter la vitesse dans la même veine à $15 \mathrm{~m} / \mathrm{s}$.

La performance essentielle du tumnel réside dans le nombre de cavitation minimal qu'on peut atteindre. La valeur minimale de ce nombre défini par $\sigma=2\left(p-p_{v}\right) / \rho V^{2}$ à partir de la pression régnant à la génératrice supérieure de la veine en charge ou à la surface libre, a été fixée à 0,02 .

On s'est imposé de pouvoir faire varier la teneur en air en l'amenant à une valeur inférieure à la teneur d'équilibre à la pression atmosphérique.

\section{Description de l'installation}

L'installation de cavitation (fig. 1) comprend la boucle hydrodynamique (groupe moteur-pompe et régulateur de vitesse, résorbeur, cuve amont, débilmètre, caisson et veine de mesure, cuve aval) et ses annexes (dégazeur, pompe à vide, compresseur, disposilifs de réglage de pression, appareils de mesure, pupitre de commande).

La cuve amont et la cuve aval ont un rôle essentiel dans le réglage de la différence de charge sous laquelle fonctionne la veine. Cette différence de charge dépend de la vitesse de rotation de la pompe et de la perte de charge d'un robinet déchargeur situé dans la cuve aval. Les différences de pression entre la cuve amont et le caisson contenant la veine ou entre le caisson et la cuve aval sont alors imposées, mais on peut faire varier les valeurs absolues de ces pressions dans les limites permises par les caractéristiques de la pompe à vide et la résistance mécanique des parois de l'installation. Le robinel déchargeur permet de choisir comme paramètres indépendants la pression dans le caisson et la pression dans la cuve aval. On peut done choisir indépendamment le nombre de cavitation et le taux de dégazage qui s'effectue dans la cuve aval et par conséquent la teneur en air d'équilibre.

\section{Groupe moteur-pompe.}

La pompe centrifuge à un étage et deux oües débite $650 \mathrm{l} / \mathrm{s}$ sous $20 \mathrm{~m}$ de charge à $1120 \mathrm{tr} / \mathrm{mn}$. Le groupe est monté sur un socle antivibratile. Il est entraîné par un moteur à courant alternatif à collecteur dont la puissance est de $165 \mathrm{~kW}$. Sa vitesse de rotation peut être réglée entre $200 \mathrm{tr} / \mathrm{mn}$ et $1200 \mathrm{tr} / \mathrm{mn}$; l'organe de réglage est un transformateur triphasé à noyau mobile télécommandé modifiant la tension du rotor.

\section{Résorbeur.}

Le résorbeur a pour mission de permettre la dissolution des petites bulles réentrainées par la pompe et d'évacuer la chaleur produite par la dissipation d'énergie dans le circuit. Il est constitué de deux conduites de grand diamètre $(900 \mathrm{~mm})$ en parallèle, de longueur $6,5 \mathrm{~m}$ disposées à la partie basse de l'installation et noyées dans la capacité de réserve des Laboratoires de $350 \mathrm{~m}^{3}$.

\section{Cuve amont.}

La cuve amont a pour ròle de déterminer d'une manière précise la charge amont. Sa section est de grand diamètre et on peut considérer que l'énergic cinétique de l'écoulement y est petite. Le réglage de la charge est déterminé par la cote de la surface libre fixée par des goulottes de déversement, et par la pression qu'on peut établir au-dessus de la surface libre à l'aide du compresseur ou de la pompe à vide. Les fluctuations du niveau sont négligeables lorsqu'on maintient le débit de surverse à une très petite valeur en ajustant la vitesse de rotation de la pompe. L'écoulement de surverse est remis en charge dans une cuve annexe avant d'être dirigé vers la cuve aval. On évite les fuites d'air dans la conduite de surverse en maintenant un niveau suffisant dans la cuve annexe à l'aide d'une vanne automatique.

\section{Débitmètre à hélice.}

Le débitmètre est disposé dans la partie rectiligne cylindrique de la colonne montante. Il comporte une hélice à caractéristique linéaire calée sur un axe en acier inoxydable. Des paliers et butées en téflon chargé au plomb (support en bronze pour les paliers) ont permis d'allier à un faible coefficient de frottement, une bonne résistance à l'usure. L'hélice envoie des impulsions électriques (soixante impulsions par tour) à un compleur chronomètre électronique; l'étalonnage du débitmètre permet de connaître le débit avec la précision de $\pm 0,8 \%$. Cet étalonnage a été effectué par la SOGREAH. Dans son installation définitive à Saint-Martind'Hères, le moyen d'essais comprendra un dispositif annexe d'étalonnage comportant un circuit de dérivation et une capacité jaugée.

\section{Coude à ailettes.}

Le tracé du coude à ailettes de $800 \mathrm{~mm}$ de diamètre qui précède immédiatement la veine a été particulièrement soigné, en vue des conditions sévères de cavitation et en vue de la réduction du sillage des ailettes.

Ce tracé résulte d'études et d'essais systématiques effectués par la SOGREAH. Le principe de la méthode de tracé consiste à découper dans un écoulement plan à potentiel des tubes de courant se raccordant à l'amont et à l'aval à des écoulements uniformes, de telle facon que ces tubes de courant présentent des survitesses locales et des gradients longitudinaux aussi réduits que possible.

On a utilisé, dans le cas présent, un réseau à potentiel dans un coude fermé à $90^{\circ}$ à parois rectilignes ayant des vitesses moyennes égales à l'entrée et à la sortie.

La ligne de courant médiane d'un tel écoulement est une ligne de jet : c'est celle qui sert à engendrer l'extra-dos de l'ailette; l'intra-dos est engendré à partir d'une ligne de courant située plus à l'extérieur, donc exempte de survitesse. 


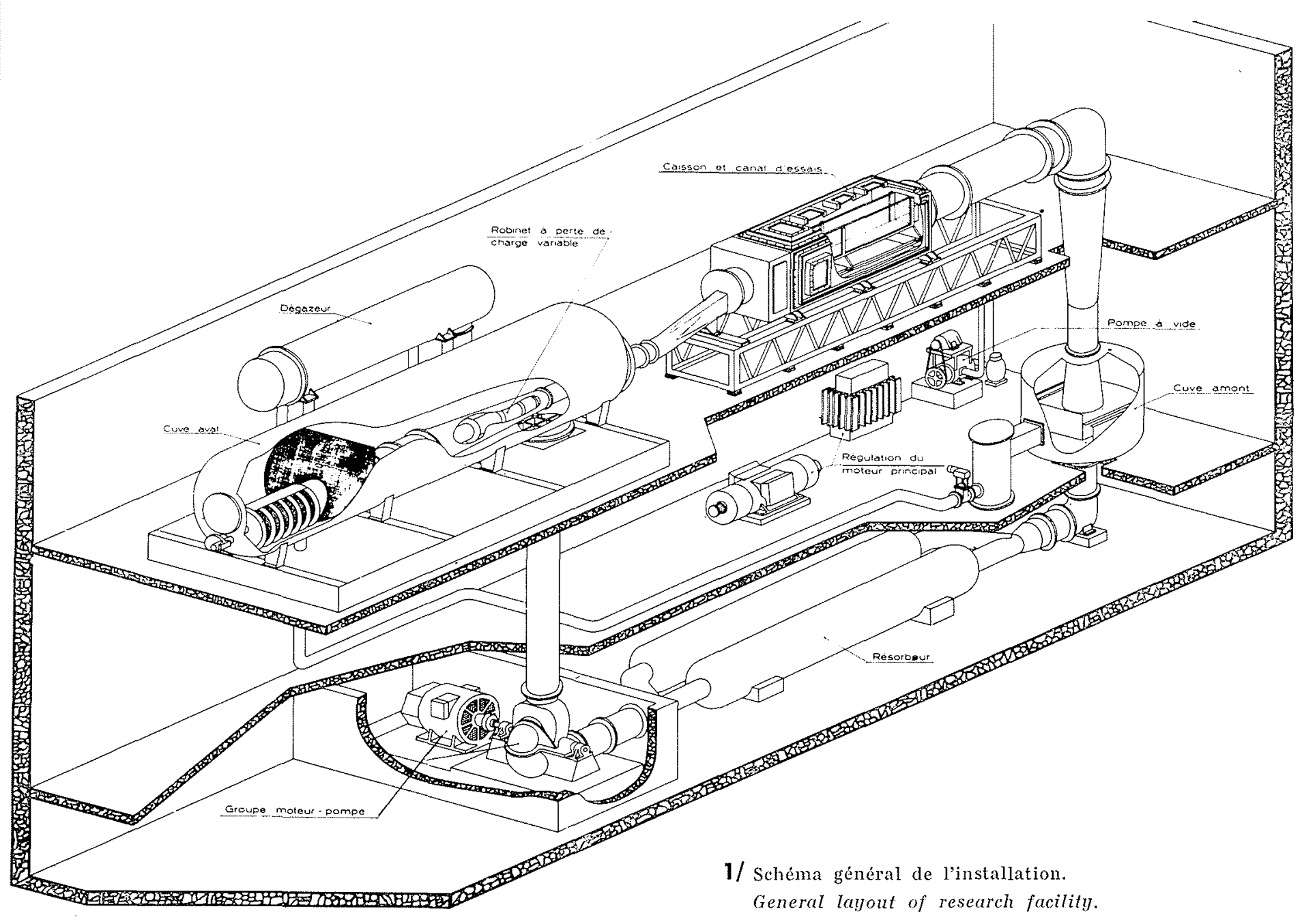

Une grille d'ailettes (seize ailettes) est logée dans une enveloppe qui a la forme d'une surface-canal engendrée par le déplacement parallèlement à ellemême d'une ellipse le long de la ligne moyenne de l'ailette. Cette surface-canal se raccorde à l'amont et à l'aval sur les tuyauteries cylindriques d'amenée et de sortie. Le coefficient de cavitation de ces coudes est moins de la moitié de celui des coudes à ailettes classiques.

\section{Convergent.}

A la sortie du coude supérieur, l'écoulement est contenu dans une conduite cylindrique horizontale de $800 \mathrm{~mm}$ de diamètre. Dans cette conduite, la vitesse moyenne pour le débit maximal ne dépasse pas $1,20 \mathrm{~m} / \mathrm{s}$. Juste avant le convergent, des prises de pressions ou des orifices d'introduction de sondes diverses sont ménagés. Ces orifices servent notamment à l'introduction d'un tube de Pitot pour l'étude de la répartition des vitesses avant le convergent. La conduite circulaire est raccordée à l'entrée rectangulaire du convergent par quatre plans inclinés à $45^{\circ}$ sur l'axe de l'écoulement. Le convergent de section rectangulaire achève la contraction de rapport global 10 à 1 . Le convergent a fait l'objet d'essais sur modèle réduit à l'échelle $1 / 2$, de manière à satisfaire aux conditions suivantes: n'ètre le siège d'aucune cavitation; donner dans le canal un écoulement à vitesse uniforme et à surface libre non perturbée.

Les essais de réception ont montré que dans la section d'entrée du canal, à l'exception d'une bande de $40 \mathrm{~mm}$ le long des parois, la vitesse est uniforme à $\pm 1 \%$ près pour la vitesse moyenne de $5,5 \mathrm{~m} / \mathrm{s}$. Dans la section du canal située à $500 \mathrm{~mm}$ de l'entrée, l'uniformité est réalisée dans les mèmes conditions à $\pm 2,7 \%$ près.

\section{Le canal de mesure.}

Actuellement, le tunnel est équipé d'un canal à surface libre à parois de verre $(175 \mathrm{~mm}$ de large et $400 \mathrm{~mm}$ de haut pour une profondeur d'eau de $280 \mathrm{~mm}$ ). Les deux parois verticales et le fond, de $16 \mathrm{~mm}$ d'épaisseur, sont montées sur un châssis métallique qui permet l'alignement. La définition géométrique de ces surfaces est assurée à $\pm 0,2 \mathrm{~mm}$ près. A cause de la grande longueur du canal $(2,5 \mathrm{~m})$ il n'a pas été possible d'éviter de disposer un étrier métallique dans la partie centrale. On a prévu la fixation des profils et le passage de conduits ou de connexions en ménageant trois trous de $20 \mathrm{~mm}$ de diamètre dans chaque paroi verticale.

L'axe du canal est incliné de $2 \mathrm{~mm}$ vers l'aval de manière que la perte de charge moyenne due au frottement sur les parois soit compensée. 


\section{Le caisson étanche (fig. 3)}

Les dimensions principales internes du caisson sont $1100 \times 1100 \times 3300 \mathrm{~mm}$. Il est concu pour résister à la dépression maximale et à une pression interne absolue de 4 bars. Il est constitué d'un cadre en cornières et de parois munies chacune de quatre hublots rectangulaires en altuglass $(300 \times$ $500 \mathrm{~mm}$ ). Pour faciliter l'accès à la veine, les deux faces latérales et la face supérieure sont entièrement démontables; les ouvertures disponibles sont de $830 \times 2450 \mathrm{~mm}$. Les faces latérales et le fond sont munis de cinq orifices prévus pour le passage de conduits ou de câbles électriques. Le caisson peut recevoir divers appareils de mesure ou d'auscultation. Un hublot circulaire de $250 \mathrm{~mm}$ de diamètre a été notamment ménagé dans sa partie supérieure pour la sortie du faisceau lumineux d'un dispositif d'investigation optique.

On a pris des précautions pour isoler le canal au point de vue des vibrations. Le caisson qui le renferme, le convergent amont et l'ouvrage de raccordement aval sont montés sur une poutre horizontale en treillis reposant sur la construction en béton par l'intermédiaire de pieds spéciaux en caoutchouc. L'ensemble de ces éléments est relié à l'amont et à l'aval au reste du circuit hydraulique par des manchons en caoutchouc.

Le caisson est normalement alimenté en air sec, de manière que la vapeur d'eau susceptible de se condenser sur les parois transparentes et de gêner l'observation, soit entraînée. Une membrane souple disposée à la partie supérieure du canal limite les projections de gouttes aux grandes vitesses et favorise l'entrainement par l'air sec, vers la cuve aval, de la vapeur d'eau formée au-dessus de la surface libre.

La remise en charge de l'écoulement à l'aval du canal est favorisée par une visicre inclinće à $30^{\circ}$ sur l'axe de l'écoulement.

Le raccordement à la cuve aval est constitué par une conduite passant progressivement de la forme rectangulaire à la forme circulaire. Cette conduite plonge vers le bas de manière à remettre en charge l'écoulement; il est important d'éviter le plus possible dans cette partie du circuit hydraulique l'entrainement d'air qui engendre perte de charge et instabilité et qui rend le débullage dans la cuve aval plus difficile.

\section{La cuve aval.}

La cuve aval fixe la charge aval par le niveau d'eau et la pression. Elle joue le rôle de vase d'expansion. Elle a des dimensions telles que la vitesse y soit assez petite pour que le débullage et une partie du dégazage puisse s'y effectuer. Enfin, elle renferme la partie de conduite dans laquelle se trouve réalisée la perte de charge additionnelle réglable. La longueur de la cuve est de $6 \mathrm{~m}$ et son diamètre de $2 \mathrm{~m}$.

La cuve est munie de six hublots d'observation. Elle renferme des tranquillisateurs en grillage métallique. Un filtre constitué d'une grille à mailles fines favorise l'ascension des très petites bulles et limite leur réaspiration par la pompe. On a supprimé la formation du vortex à l'entonnement de la conduite en direction de la pompe en disposant un radeau près de la surface libre et un croisillon dans la conduite.

La perte de charge additionnelle est réalisée par un robinet spécial qui comporte essentiellement un corps conique à l'intérieur duquel peut se déplacer suivant l'axe un arbre muni de disques nérie, de diamètres différents, et tels que chacun engendre une perte de charge liée à sa position.

La répartition et le nombre de ces disques résultent d'une optimisation relative à leur comportement à la cavitation de mélange.

Les cuves aval et amont sont équipées de soupapes de mise automatique à l'atmosphère en cas de défaut de tension électrique, pour éviter les détériorations consécutives au coup de bélier éventuel

\section{Circuit d'air comprimé et de vide.}

La cuve amont, le caisson et la cuve aval peuvent ètre reliés soit au circuit de vide, soit au circuit d'air comprimé. Chacune de ces enceintes peut être reliée à l'atmosphère prise comme référence par des vannes à fuite réglable, télécommandées. Ia pompe à vide est une pompe à anneau liquide (puissance : $8,8 \mathrm{~kW}$, débit : $372 \mathrm{~m}^{3} / \mathrm{h}$, pression minimale : 15 mbars). Le compresseur a un débit nominal de $120 \mathrm{~m}^{3} / \mathrm{h}$. A la sortie du réservoir $\left(1,8 \mathrm{~m}^{3}\right)$ l'air passe dans un filtre à huile. L'air destiné au caisson traverse un élément dessiccateur au sulfate de calcium.

\section{Le dégazeur.}

Le dégazeur est un bouilleur sous vide à température ambiante. Il comprend essentiellement de longs canaux horizontaux à faible profondeur (surface libre totale : $16,7 \mathrm{~m}^{2}$ ) disposés dans une chambre cylindrique de $1000 \mathrm{~mm}$ de diamètre el de $4,4 \mathrm{~m}$ de longueur reliée à la pompe à vide; la naissance des bulles est favorisée à l'amont des canaux par la formation de jets cavitants. A l'aval, la remise en charge s'effectue dans une conduite verticale. Le niveau de l'eau dans cette conduite est réglé par une vanne automatique à commande hydraulique agissant sur le débit à l'entrée du dégazeur. Le dégazeur peut fonctionner de différentes manières, soit au remplissage de l'installation, soit en dérivation pendant le fonctionnement. Dans tous les cas, une pompe d'injection multiétagée à l'aval du dégazeur introduit l'eau dans le tunnel à l'amont de la pompe principale avec un débit maximal de $15 \mathrm{l} / \mathrm{s}$. Au remplissage on peut atteindre la teneur de $6,5 \mathrm{mg}$ d'air par litre.

\section{Le pupitre de commande.}

Le pupitre de commande rassemble les commandes de démarrage et d'arrêt du moteur principal el des organes annexes, les commandes de réglage de vitesse de la pompe principale, de la position du robinet déchargeur, et les télécommandes des vannes à fuite de mise à l'atmosphère. On y a disposé aussi les appareils de mesure des paramètres suivants : vitesse de la pompe principale, tension et intensité du moteur, pression dans la cuve amont. niveau de surverse dans cette cuve, pression dans le caisson, pression dans la cuve aval, pression du compresseur. 


\section{2/}

Vue d'ensemble de l'étage supérieur de l'installation.

General view of the upper level.

\section{3/}

Vue de l'intérieur du caisson. On distingue, à l'amont du canal, le support de profil et le tuyau de ventilation de la cavité et, à droite, le chariot support d'un ensemble optique.

View inside the observation chamber. The test model mounting and vent pipe can be seen at the upstream flume end and an optical instrument carriage on the right.
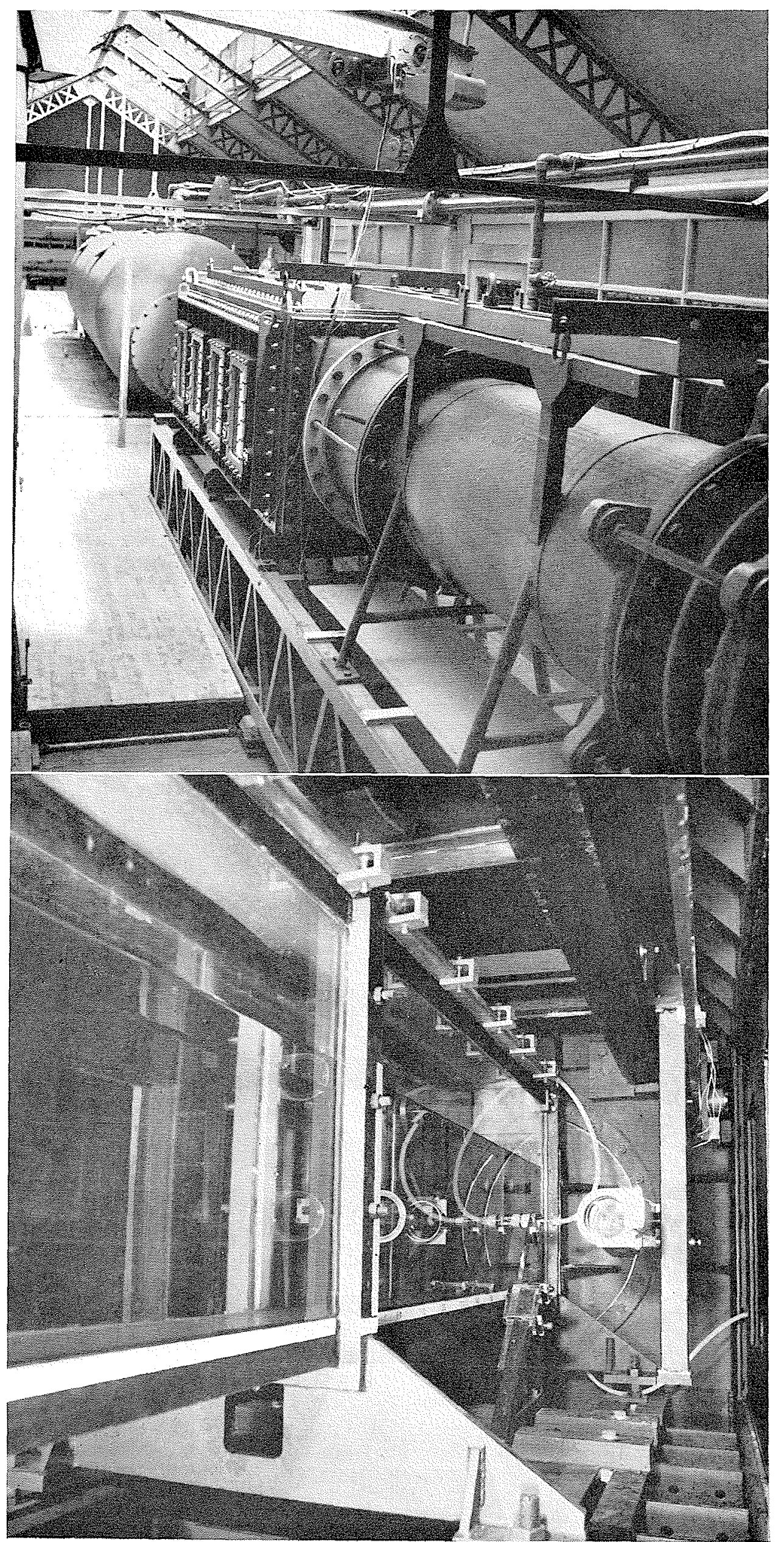
Réglage et stabilité.

Le dimensionnement des principaux organes de la boucle et en particulier des cuves amont et aval confère à l'installation d'essai une très bonne stabilité.

Le réglage des conditions de fonctionnement de la boucle concerne trois paramètres principaux :

- le débit est réglé par variation de la vitesse de la pompe, les fluctuations du débit étant filtrées par déversement dans les goulottes situées à l'intérieur de la cuve amont;

- les pressions d'air sont réglées par l'action des vannes à fuite de mise à l'atmosphère de chacun des éléments, ceux-ci étant reliés soit au circuit d'air comprimé, soit au circuit de vide;

- la teneur en air est réglée par l'action du vide dans la cuve aval et, en particulier, par l'action du dégazeur lors du remplissage de l'installation.

Par ailleurs, le réglage du niveau de reprise à l'aval des goulottes de la cuve amont est réalisé par un robinet-vanne commandé par un régulateur électronique à action proportionnelle.
Tel qu'il se présente, le tunnel hydrodynamique des Laboratoires de Mécanique des Fluides de l'Université de Grenoble est adapté aux recherches sur l'hydrodynamique des profils cavitants ou supercavitants. Par les hublots ménagés dans les parois du caisson, il offre des possibilités variées d'observation visuelle ou photographicque, soit pour la cavitation naissante, soit pour la cavitation développée. Un dispositif optique à fort grandissement destiné à l'observation détaillée du champ d'écoulement autour des profils est actuellement en cours de mise au point.

L'équipement du tunnel permet de régler la teneur en air. Les dimensions du caisson sont assez grandes pour qu'on puisse y placer les dispositifs de mesures dynamiques globales tels que balances à plusieurs composantes.

Le dispositif de débullage puissant constitué par la cuve aval permet de réaliser des cavités ventilées. On peut utiliser les accès ménagés dans les parois pour la traversée des conduits de mesure de pressions locales ou de pressions de cavité.

Les dimensions du tunnel, ses performances et ses équipements en font un outil de recherche particulièrement intéressant dans sa catégorie.

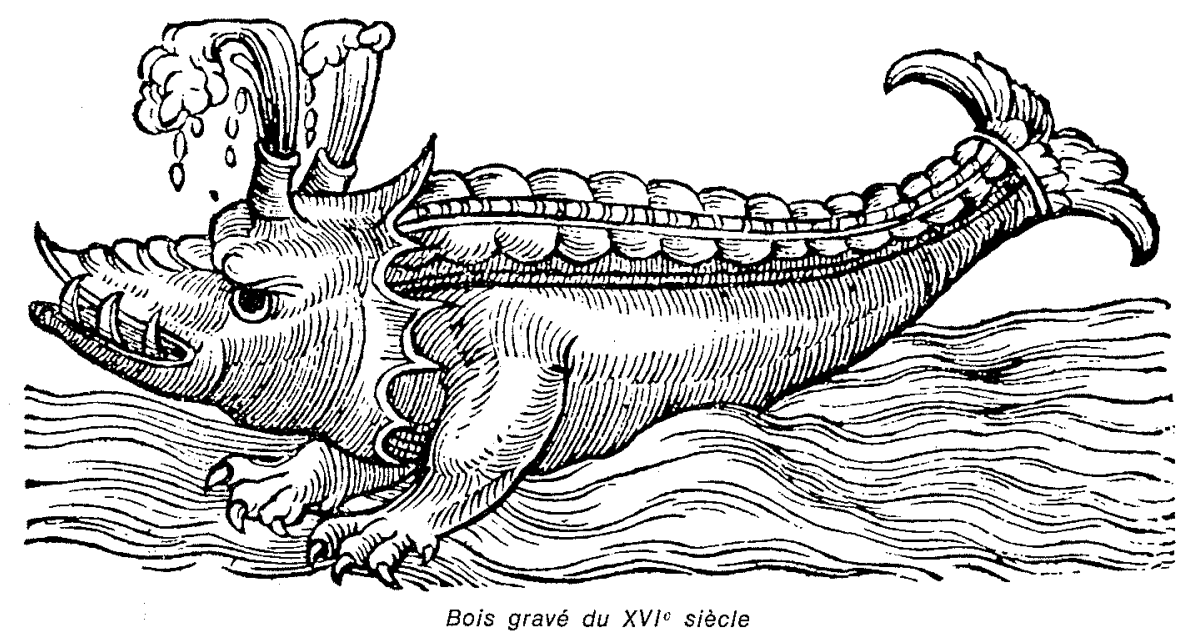

